\title{
Structural Results on Optimal Rate and Number of Clusters in Cluster based Cooperative MIMO Sensor Networks
}

\author{
Laxminarayana S Pillutla and Vikram Krishnamurthy \\ Department of Electrical and Computer Engineering \\ 2332 Main Mall, The University of British Columbia \\ Vancouver, BC V6T1Z4, Canada. \\ E-mail:\{laxp, vikramk\}@ece.ubc.ca
}

\begin{abstract}
We present structural results on optimal number of clusters and optimal rate with respect to long haul distance in a cluster based cooperative multiple input and multiple output (MIMO) sensor network. Using the concepts of super and sub modular functions and their associated properties it has been proved that the number of clusters to minimize the energy consumption is a decreasing function of long haul distance. From this, we conclude that as the long haul distance increases the cooperation among the nodes increases to counter the path loss due to fading in the channel. Furthermore, we also prove the rate to minimize the energy consumption is a decreasing function of long haul distance. Finally, through our numerical results we observe that optimizing node cooperation can lead to large scale energy savings.
\end{abstract}

\section{INTRODUCTION}

Sensor networks have been at the forefront of research in recent years owing to the recent hardware advances which allow more signal processing functionalities to be integrated into a single device that is as small as a piece of grain, and which can be used as a fully functional wireless sensor node. Such sensor nodes are expected to operate on small batteries for long periods of time, as replacement can be difficult if not impossible. Consequently, minimizing energy consumption is an important design consideration for sensor networks. As an effort in this direction cooperation based information transmission schemes have been proposed for sensor networks in [1] and [2].

In [1] it has been shown that by incorporating cooperation among the nodes based on the Alamouti code [3], it is possible to reduce energy consumption as well as transmission delay over some distance ranges. Another MIMO architecture based on the well-known V-BLAST (Bell Labs Layered Space-time) architecture had been proposed for sensor networks in [2]. Unlike the Alamouti code based information transmission scheme proposed in [1], this type of architecture does not require joint encoding on the transmit side. This eliminates the local processing and local communication steps involved in the architecture proposed in [1].

This work was supported by a research grant from Natural Sciences and Engineering Research Council (NSERC) of Canada
For the case of dense micro-sensor networks an application specific protocol called low-energy adaptive clustering hierarchy $(L E A C H)$ had been proposed in [4]. In LEACH, all the nodes would transmit the data to a clustering head $(\mathrm{CH})$, which in turn would relay the data to the base station (BS). Acting as a $\mathrm{CH}$ could be quiet energy intensive, hence LEACH employs randomized rotation of $\mathrm{CH}$. It needs to be noted that $\mathrm{LEACH}$ does not employ node cooperation within the clusters.

In this paper, we study the impact of clustering along with the cooperative MIMO techniques proposed in [1] on the energy efficiency of large scale sensor networks. The main contributions of our work are as follows:

1) By proving the total energy consumption $\left(E_{\text {total }}\right)$ to satisfy increasing differences [5], [6] with respect to number of clusters, we prove optimal number of clusters as a decreasing function of distance. By virtue of this result, we conclude that as the distance increases the cooperation among nodes increases, thereby increasing the diversity within the cluster to counter the fading in the channel.

2) In [1], to improve the energy efficiency of sensor networks (especially for short distances) rate optimized cooperative MIMO transmission scheme was proposed. In this paper, by proving $E_{\text {total }}$ to satisfy increasing differences with respect to rate, we prove the optimal rate as a decreasing function of distance. This intuitively suggests that, for large distances as the fading in the channel increases, the optimal rate that achieves the desired probability of error decreases gradually.

3) Using our numerical results it was observed that for long distances when probability of error desired is small, employing a cooperative transmission in which the nodes with in the cluster are optimized can lead to large scale energy savings (even at the expense of bandwidth).

4) Also for a large scale sensor network allowing all the nodes to cooperate in the long haul transmission may not lead to large scale energy savings, because the increased energy for local communication may offset the diversity 
advantage obtained due to cooperative transmission.

The rest of this paper is organized as follows: In Section II, we present system model description and assumptions. In Section III, we derive the energy consumption in a cluster based cooperative MIMO sensor network. Section IV contains structural results with respect to optimal number of clusters and optimal rate. Section V contains numerical results and Section VI contains some important conclusions of this work.

\section{System Model AND Assumptions}

\section{A. System Model of Cluster Based Cooperative MIMO Trans- mission Scheme}

We consider the scenario where a fixed number $(N)$ of homogeneous sensors called nodes are dropped to keep track of an event of interest. These nodes are divided into small equal groups called clusters. The nodes within each cluster, collaborate to form a virtual multiple antenna system and transmit information to an access point (AP) (for example, a base station) using the space time block codes (STBC) [7].

\section{B. Assumptions}

To simplify our analysis, as in [1], we neglect the energy consumption for base band processing (comprising of source coding, channel coding and digital modulation). We also assume the nodes are capable of operating in multiple modes namely: active, transient and sleep. Operating the nodes on a multi-mode basis can lead to significant energy savings [8]. Since the power loss in sleep and transient modes is small, hence as in [1] we neglect them in our analysis.

The channel for local communication is assumed to be time invariant additive white Gaussian noise (AWGN) channel with square law path loss as in [1]. But for long haul transmissions, we assume the channel to be of Rayleigh fading type with square law path loss and AWGN as the background noise.

As in [1] and [8], we consider circuit energy consumption along with transmission energy consumption for our analysis. This is done because for short distances, by deploying large constellation sizes circuit energy consumption can be reduced significantly [1].

We assume $N$ sensors are dropped uniformly in a square area $^{1}$ of side $M$ to keep track of an event of interest. Let these $N$ sensors be divided into $K$ balanced clusters so that each cluster has $\left\lfloor\frac{N}{K}\right\rfloor$ nodes where $\lfloor X\rfloor$ denotes $X$ rounded down to the nearest positive integer. For convenience, from here on, we use $\frac{N}{K}$ instead of $\left\lfloor\frac{N}{K}\right\rfloor$. The clusters are assumed balanced to ensure the life of all clusters remains the same, which is not true when the clusters are unbalanced (i.e. when they have unequal number of nodes) as the cluster with less number of nodes may die out quickly. Further, we assume the sensors to be of the same distance $(D)$ from the AP (this implies the clusters containing this sensors also have the same distance from the AP) and each of them have $L$ bits to

\footnotetext{
${ }^{1}$ We assume square area purely for notational convenience. The results in the paper hold (with appropriate modification) for sensors uniformly distributed on an arbitrary region in $\mathbb{R}^{2}$ as long as the area of the region is known.
}

transmit. Further all the clusters containing the sensor nodes are assumed identical, and the distribution of nodes with in the cluster is assumed to be uniform. Since the clusters are identical and the distance of all the clusters from the access point is the same, it is reasonable to expect that all the clusters employ same rate $(b)$ for transmission.

Next, we assume the clusters are elliptical in shape, however other uniform shapes such as square, circle can be assumed as long as the assumption of uniform distribution of nodes with in the cluster can be satisfied. For example in [4], the clusters are assumed to be circular. In our case they are assumed elliptical, since an ellipse is more general compared to a circle and can model sensors in a line.

For analysis throughout this paper, we assume M- ary quadrature amplitude modulation (MQAM) as the underlying modulation scheme. To reduce the burden on sensor nodes, we assume the clustering algorithm is implemented by the access point.

\section{Energy CONSUmption In Cluster BASED COOPERATIVE MIMO SENSOR NETWORK}

In this section, we derive an expression (strictly speaking an upper bound) for the total energy consumption of the cluster based cooperative MIMO transmission scheme.

Denoting $E_{\text {cluster }}$ as the energy consumption in a cluster, we have

$$
E_{\text {cluster }}=E_{\text {local }}+E_{\mathrm{LH}},
$$

where $E_{\text {local }}$ and $E_{\mathrm{LH}}$ denote the energy consumption for local and long haul transmission.

Considering the circuit and transmission energy costs separately, $E_{\text {local }}$ and $E_{\mathrm{LH}}$ can be expressed as

$$
\begin{aligned}
E_{\text {local }} & =E_{\text {c-local }}+E_{\text {Tx-local }} \\
E_{\mathrm{LH}} & =E_{\mathrm{c}-\mathrm{LH}}+E_{\mathrm{Tx}-\mathrm{LH}},
\end{aligned}
$$

where $E_{\text {c-local }}, E_{\text {Tx-local }}, E_{\text {c-LH }}$ and $E_{\text {Tx-LH }}$ are the local circuit, local transmission, long haul circuit and long haul transmission energy costs.

Denoting $P_{\mathrm{T}}$ and $P_{\mathrm{R}}$ as the power consumption in the transmitter and receiver circuits, we can write

$$
\begin{aligned}
& P_{\mathrm{T}}=P_{\mathrm{DAC}}+P_{\mathrm{mix}}+P_{\mathrm{filtx}} \\
& P_{\mathrm{R}}=P_{\mathrm{LNA}}+P_{\mathrm{mix}}+P_{\mathrm{IFA}}+P_{\text {filrx }}+P_{\mathrm{ADC}},
\end{aligned}
$$

where $P_{\mathrm{DAC}}, P_{\mathrm{mix}}, P_{\mathrm{filtx}}, P_{\mathrm{LNA}}, P_{\mathrm{IFA}}, P_{\text {filrx }}$ and $P_{\mathrm{ADC}}$ are the power consumption values in digital to analog converter (DAC), mixer circuit, transmitter filter, low noise amplifier (LNA), intermediate frequency amplifier (IFA), receiver filter and analog to digital converter.

Further, if we denote $T_{\text {on }}$ as the ON time of the nodes then

$$
T_{\mathrm{on}} \approx \frac{L}{B b},
$$

where $b$ as defined before denotes the rate (or constellation size) and $B$ denotes the modulation bandwidth [1]. 
A. Energy consumption for local information flow ( $\left.E_{l o c a l}\right)$ within a cluster

In this subsection, we calculate the energy consumption for local information flow $\left(E_{\text {local }}\right)$ per cluster defined in (2). Since during local information flow, there are always $\left(\frac{N}{K}-1\right)$ nodes listening (note that each cluster has $\frac{N}{K}$ nodes), hence the total circuit energy per bit for local information flow can be written as

$$
\begin{aligned}
E_{\text {c-local }} & =\frac{N}{K}\left(P_{\mathrm{T}}+\left(\frac{N}{K}-1\right) P_{\mathrm{R}}\right) \frac{T_{\text {on }}}{L} \\
& =\frac{N}{K B b}\left(P_{\mathrm{T}}+\left(\frac{N}{K}-1\right) P_{\mathrm{R}}\right) .
\end{aligned}
$$

Next, we consider the transmission energy required for the local information flow. For our future use, we define

$$
Q(x)=\frac{1}{\sqrt{2 \pi}} \int_{x}^{\infty} e^{-\frac{y^{2}}{2}} d y .
$$

Since we are assuming a square law path loss model, hence the energy consumption per bit for local information flow can be written as [9] [1]

$$
E_{\text {Tx-local }}=(1+\alpha) E_{b-a w g n} \frac{(4 \pi)^{2} M_{l} N_{f}}{G_{t} G_{r} \lambda^{2}} \sum_{i=1}^{\frac{N}{K}} \sum_{j=1, j \neq i}^{\frac{N}{K}} d_{i j}^{2},
$$

where

$$
1+\alpha=\frac{3}{\eta}\left[\frac{2^{\frac{b}{2}}-1}{2^{\frac{b}{2}}+1}\right],
$$

$\eta$ is the drain efficiency of the power amplifier [1], $E_{b-a w g n}$ is the per bit energy consumption in an AWGN channel and can be calculated using Chernoff bound on the probability of error expression for MQAM provided in [9] (as in [8]), $M_{l}$ is the link margin, $N_{f}$ is the noise figure, $G_{t} G_{r}$ is the combined transmitter and receiver gain and $d_{i j}$ is the distance between $i$ th and $j$ th nodes respectively.

As in [8] $E_{b-a w g n}$ can be written as

$$
E_{b-a w g n}=\frac{2}{3}\left(\frac{2^{b}-1}{b}\right) N_{0} \ln \left(\frac{2}{P_{b}}\right) .
$$

Next since the clusters are assumed elliptical, hence we obtain square of the maximum distance of the cluster $\left(d_{\max }^{2}\right)$ to be

$$
d_{\max }^{2}=\frac{M^{2}\left(c^{2}+1\right)}{\pi K c} .
$$

Now we re-write (7) using (9) and (10) (by replacing $d_{i j}^{2}$ with $\left.d_{\max }^{2}\right)$, and thus obtain

$$
E_{\text {Tx-local }} \leq \frac{N}{K^{2}}\left(\frac{N}{K}-1\right) \frac{\left(2^{\frac{b}{2}}-1\right)^{2}}{b} M^{2} \epsilon_{\text {local }},
$$

where we define $\epsilon_{\text {local }}=\frac{2}{\eta} \frac{(4 \pi)^{2} M_{l} N_{f} N_{0} \ln \left(\frac{2}{P_{b}}\right)}{G_{t} G_{r} \lambda^{2}}\left(\frac{c^{2}+1}{\pi c}\right)$.
Thus, the maximum total energy required to transmit $L$ bits locally after approximating $E_{\text {Tx-local }}$ as an equality is

$$
\begin{aligned}
& E_{\text {local }}=\frac{N L}{K B b}\left(P_{\mathrm{T}}+\left(\frac{N}{K}-1\right) P_{\mathrm{R}}\right)+\frac{N}{K^{2}} \\
& \left(\frac{N}{K}-1\right) \frac{\left(2^{\frac{b}{2}}-1\right)^{2}}{b} \epsilon_{\text {local }} M^{2} L .
\end{aligned}
$$

\section{B. Energy consumption in the long haul $\left(E_{L H}\right)$ per cluster}

In this subsection, we calculate the energy consumption in the long haul $\left(E_{\mathrm{LH}}\right)$ per cluster defined in (2).

Since in long haul all the $\frac{N}{K}$ nodes with in a given cluster transmit cooperatively, the per bit circuit energy consumption for long haul can be written in terms of $T_{\text {on }}$ and spatial rate $(r)$ of STBC as

$$
E_{C-L H}=\left(\frac{N}{K} P_{\mathrm{T}}+P_{R}\right) \frac{T_{\text {on }}}{L r} .
$$

Next we calculate the per bit transmission energy consumption in the long haul $\left(E_{\mathrm{Tx}-\mathrm{LH}}\right)$.

In general for a MQAM system with square constellations, the average probability of error $\left(P_{b}\right)$ in Rayleigh fading with $N_{T}$ transmit antennas and single receive antenna (i.e. a multiple input and single output (MISO) system) employing STBC with spatial rate $(r)$ is given approximately as

$$
P_{b} \approx \mathbb{E}_{\gamma}\left[\frac{4}{b}\left(1-\frac{1}{2^{\frac{b}{2}}}\right) Q\left(\sqrt{\frac{3 b}{2^{b}-1} \gamma}\right)\right],
$$

where $Q(x)$ is defined as in (6), $\mathbb{E}_{\gamma}$ denotes the expectation operator with respect to the random variable $\gamma$, which corresponds to the instantaneous received signal to noise ratio $(\mathrm{SNR})^{2}$ and is defined as

$$
\gamma=\sum_{i=1}^{N_{T}}\left|\mathbf{h}_{i 1}\right|^{2} \frac{r E_{b}}{N_{T} N_{0}},
$$

where $\mathbf{h}_{i 1}$ is the channel gain between the $i$ th transmit antenna and the single receive antenna, $E_{b}$ indicates the per bit total transmission energy, $r(\leq 1)$ denotes the spatial rate of the STBC used and $\frac{N_{0}}{2}$ denotes the noise variance per dimension. The factor of $\frac{1}{N_{T}}$ in (15) accounts for the equal power split between the $N_{T}$ transmitter antennas.

Using (14) and (15) and the approximations in [1], the energy required to transmit one bit over the $\mathrm{LH}$ can be written as

$E_{\mathrm{b}-\mathrm{Tx}} \leq \frac{2}{\eta}\left(\frac{4}{P_{b}}\right)^{\frac{1}{N_{T}}} \frac{\left(2^{\frac{b}{2}}-1\right)^{2}}{b^{\frac{1}{N_{T}}}+1} \frac{1}{r} N_{T} N_{0} \frac{(4 \pi D)^{2}}{G_{t} G_{r} \lambda^{2}} M_{l} N_{f}$,

where $\eta$ is the drain efficiency of the power amplifier.

Observing that each cluster with $\frac{N}{K}$ nodes and the access point (AP) constitute an MISO system, we can upper bound

\footnotetext{
${ }^{2}$ The SNR is defined as in (15) to account for the increase in noise power because of bandwidth expansion that arises due to using a STBC with rate $r$.
} When $r=1$, the definition in (15). is same as the one in [1] 
the transmission energy for a single cluster $E_{\mathrm{Tx}-\mathrm{LH}}$ per bit as (by replacing $N_{T}$ with $\frac{N}{K}$ )

$$
E_{\mathrm{Tx}-\mathrm{LH}} \leq \frac{N}{K} \frac{\left(2^{\frac{b}{2}}-1\right)^{2}}{b^{\frac{K}{N}+1}}\left(\frac{4}{P_{b}}\right)^{\frac{K}{N}}\left(\frac{1}{r}\right) \epsilon_{\mathrm{LH}} D^{2},
$$

where we define $\epsilon_{\mathrm{LH}}=\frac{2}{\eta} \frac{N_{0}(4 \pi)^{2} M_{l} N_{f}}{G_{t} G_{r} \lambda^{2}}$. Note that in deriving (17), we assumed that the long haul distance $D$ is same for all the nodes in the cluster.

Because of local transmission required for node cooperation, each node in the cluster has $\frac{N L}{K}$ bits to transmit (i.e. each node has its data along with the remaining $\left(\frac{N}{K}-1\right)$ nodes data), thus the total number of bits to transmit in the long haul is equal to $\frac{N^{2} L}{K^{2}}$. Approximating the upper bound in (17) as an equality the total energy (circuit plus transmission energy) required to transmit $L$ bits of each node in the cluster cooperatively in the long haul is equal to

$$
\begin{aligned}
& E_{\mathrm{LH}}=\left(\frac{N}{K}\right)^{2} \frac{L}{b B r}\left[\frac{N}{K} P_{T}+P_{R}\right]+\left(\frac{N}{K}\right)^{3} \frac{\left(2^{\frac{b}{2}}-1\right)^{2}}{b^{\frac{K}{N}}+1} \\
& \left(\frac{4}{P_{b}}\right)^{\frac{K}{N}}\left(\frac{1}{r}\right) \epsilon_{\mathrm{LH}} D^{2} L .
\end{aligned}
$$

C. Total Energy consumption $\left(E_{\text {total }}\right)$ for cluster based cooperative MIMO transmission scheme

In this subsection, we calculate the maximum total energy required for the cluster based cooperative MIMO scheme. Using (1), (12) and (18), the maximum total energy consumption $\left(E_{\text {total }}\right)$ for the cooperative MIMO scheme is (clusters are assumed identical)

$$
\begin{aligned}
& E_{\text {total }}=K E_{\text {cluster }} \\
& =\frac{N L}{b B}\left[P_{\mathrm{T}}\left(\frac{N^{2}}{K^{2} r}+1\right)+\left(\frac{N}{K}+\frac{N}{K r}-1\right) P_{\mathrm{R}}\right] \\
& +\frac{N}{K}\left(\frac{N}{K}-1\right) \frac{\left(2^{\frac{b}{2}}-1\right)^{2}}{b} M^{2} L \epsilon_{\text {local }} \\
& +\frac{N^{3}}{K^{2}} \frac{\left(2^{\frac{b}{2}}-1\right)^{2}}{b}\left(\frac{4}{b P_{b}}\right)^{\frac{K}{N}}\left(\frac{1}{r}\right) \epsilon_{L H} D^{2} L .
\end{aligned}
$$

\section{STRUCTURAL RESULTS ON OPTIMAL NUMBER OF CLUSTERS $\left(K^{*}\right)$ AND OPTIMAL RATE $\left(b^{*}\right)$}

In this subsection, we prove important structural results on optimal number of clusters $\left(K^{*}\right)$ and optimal rate $\left(b^{*}\right)$ with respect to the long haul distance $D$.

Before we proceed to the structural results of the paper we provide a brief background on supermodular functions and their associated properties [5] and [6].

Definition 1: A continuous function $F(s, a)$ is said to be supermodular if $\frac{\partial^{2} F(s, a)}{\partial a \partial s} \geq 0$.

We now state the following simplified version of Topkis's Monotonicity theorem in [5]. This simplified version provided in [6] is adequate to prove the structural results in our paper.
Lemma 1: For the optimization problem below:

$$
\text { minimize } F(s, a) \text { subject to } s \in S
$$

Suppose that $S \subset \mathbb{R}$ forms a sublattice on $\mathbb{R}$ and has atleast one solution for each $a \in A$. Suppose also that $F$ satisfies (strictly) increasing differences in $(s, a)$. Then optimal $s$ is always decreasing in a.

A. Structural Results on Optimal Number of Clusters $K^{*}$ with respect to Long Haul Distance (D)

In this subsection, we prove that the optimal solution $K^{*}$ obtained for the following optimization problem in (20) and (21) is a decreasing function of long haul distance $(D)$. By virtue of this, we conclude that as the long haul distance $(D)$ increases, the cooperation among the nodes increases.

For our further analysis we state the following optimization problem:

$$
\begin{array}{cl}
\underset{K}{\operatorname{minimize}} & E_{\text {total }}(b, K, D) \\
& \text { Subject to: } 1 \leq K \leq N,
\end{array}
$$

where the constraint in (21) ensures that $K^{*}$ is greater than or equal to one and less than or equal to $N$ and $E_{\text {total }}(K, D)$ is same as the expression in (19). We are now ready to state the following theorem:

Theorem 1: For the energy optimization problem in (20), subject to the constraint (21), for fixed number of sensors $(N)$ and given probability of error $\left(P_{b}\right)$, the optimal number of clusters $K^{*}$ is a decreasing function of the long haul distance $D, \forall D \in\left[D_{\min }, D_{\max }\right]$, where $D_{\min }$ satisfies the inequality $K D_{\min }>>\frac{M^{2}\left(c^{2}+1\right)}{4 \pi c}$ and $D_{\max }<\infty$.

Proof: See [10] for details.

B. Optimization Problem for Rate and Structural Results for the Optimal Rate $\left(b^{*}\right)$

In this subsection, we formulate the optimization problem for rate and prove that the optimal rate $\left(b^{*}\right)$ is a decreasing function of long haul distance $(D)^{3}$. The optimization problem for rate $(b)$ is formulated as follows:

$$
\begin{array}{ll}
\underset{b}{\operatorname{minimize}} & E_{\text {total }}(b, D) \\
& \text { Subject to: } 2 \leq b \leq b_{\max },
\end{array}
$$

where $E_{\text {total }}(b, D)$ is same as the expression in (19) and $b_{\max }$ is determined subject to the peak power constraint of each node. The value of $b_{\max }$ is taken as equal to 12 for all the numerical results

Theorem 2: For the energy optimization problem in (22), subject to the constraint in (23), for given number of clusters $(K)$ and probability of error $\left(P_{b}\right)$ the optimal rate $b^{*}$ is a decreasing function of long haul distance $D, \forall D \in\left[D_{\min }, D_{\max }\right]$ where $D_{\min }$ satisfies the inequality $K D_{\min }>>\frac{M^{2}\left(c^{2}+1\right)}{4 \pi c}$ and $D_{\max }<\infty$.

Proof: See [10] for details.

\footnotetext{
${ }^{3}$ The rate optimization for sensor networks was first proposed in [1], here we provide an analytical result on the variation of $b^{*}$ with $D$ (see Section I for details).
} 


\section{NumERICAL RESUlTS}

For our numerical results we use the system parameter values in [1]. To calculate the values of $P_{\mathrm{ADC}}$ and $P_{\mathrm{DAC}}$, we used the model developed in the Appendix of [8]. The value of $P_{\mathrm{IFA}}$ is also taken from [8].

Figure (1) shows the comparison of energy consumption between the cases when all the clusters have two cooperating nodes to that of when the number of nodes with in the cluster are optimized.

The Fig. (1) ( top and bottom) indicates that for the case when number of cooperating nodes with in the cluster are equal to two the rate optimized and unoptimized schemes have almost the same energy consumption for large distances. This is to be expected, since in the long haul the optimal rate is equal to two, which is same as the rate in the unoptimized scheme. However a close observation of the figure reveals that for short distances (i.e. between 0 to $30 \mathrm{~m}$ ) the rate optimized scheme may be more energy efficient because of the reduction in circuit energy consumption due to deploying of higher constellation sizes. But when compared to the cluster optimized scheme both the schemes perform better for the case when probability of error $\left(P_{b}\right)$ equal to $10^{-4}$ (top figure) except for a small window between 250 to $300 \mathrm{~m}$. However the situation changes dramatically when $P_{b}$ value is reduced to $10^{-5}$ (bottom figure). For this case the rate optimized and unoptimized schemes perform worse (except for a small window between 0 to $100 \mathrm{~m}$ ) compared to the cluster optimized scheme.

In Fig. (2), we prove that it may not be judicious to allow all the nodes cooperate in the long haul. The figure shows that in the long haul when all the nodes cooperate the energy consumption increases drastically compared to the case when the nodes with in the cluster are optimized. This could be attributed to the fact that when all the nodes cooperate the energy consumption for local information circulation might be more compared to the reduction in energy obtained due to cooperative transmission. For this plot the value of probability of error $\left(P_{b}\right)$ was set equal to $10^{-4}$.

\section{CONCLUSIONS}

We considered energy efficiency analysis of cluster based cooperative MIMO sensor networks. Using an expression for total energy consumption, we proved that the optimal number of clusters is a decreasing function of long haul distance, thereby proving that as the long haul distance increases, the cooperation among the nodes increases, in order to attain the desired probability of error. Further, we proved the optimal rate as a decreasing function of long haul distance. Next, through our numerical results, we observe that for high and moderate values of probability of error for most distances, it is energy efficient to employ two nodes per cluster than optimizing the number of nodes with in the cluster. However when the probability of error desired is small, optimizing the number of nodes with in the cluster can lead to large scale energy savings. Finally optimizing the number of nodes with

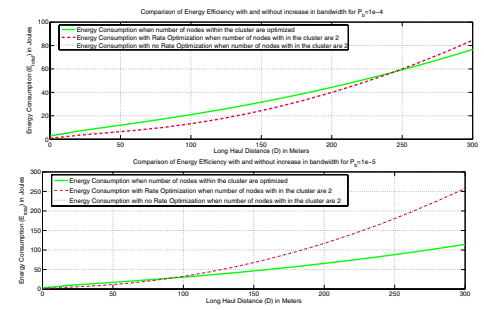

Fig. 1. The top figure shows the comparison of optimized total energy consumption versus long haul distance for $P_{b}=10^{-4}$ when the number of nodes with in the cluster are equal to two (with and without rate optimization) and when the number of nodes with in the cluster are optimized. The bottom figure shows the same scenario when $P_{b}=10^{-5}$.

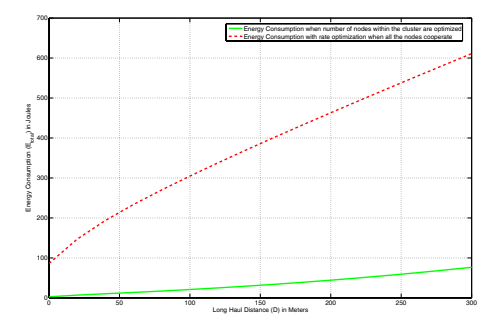

Fig. 2. The figure shows the comparison of optimized total energy consumption versus long haul distance when all the sensor nodes cooperate with rate optimization to that of the case when the number of nodes with in the cluster are optimized for $P_{b}=10^{-4}$.

in the cluster can lead to large scale energy savings compared to the case when all the nodes cooperate.

\section{REFERENCES}

[1] S. Cui, A. J. Goldsmith, and A. Bahai, "Energy-efficiency of MIMO and Cooperative MIMO Techniques in Sensor Networks," IEEE Journal on Selected Areas in Communications, vol. 22, no. 6, pp. 1089-1098, Aug. 2004.

[2] S. K. Jayaweera, "An Energy-efficient Virtual MIMO Communications Architecture Based on V-BLAST Processing for Distributed Wireless Sensor Networks," in Proc. IEEE SECON, Nov. 2004.

[3] S. M. Alamouti, "A Simple Transmit Diversity Technique for Wireless Communications," IEEE Journal on Selected Areas in Communications, vol. 16 , no. 8 , pp. 1451-1458, Oct. 1998.

[4] W. B. Heinzelman, A. P. Chandrakasan, and H. Balakrishnan, "An Application-Specific Protocol Architecture for Wireless Microsensor Networks," IEEE Transactions on Wireless Communications, vol. 1, no. 4, pp. 660-670, Oct. 2002.

[5] D. M. Topkis, Supermodularity and Complementarity, Princeton University Press, New Jersey, 1998.

[6] R. Amir, "Supermodularity and Complementarity in Economics: An Elementary Survey," Southern Economic Journal, vol. 71, no. 3, pp. 636-660, Jan. 2005.

[7] V. Tarokh, H. Jafarkhani, and A. R. Calderbank, "Space-Time Block Codes from Orthogonal Designs," IEEE Transactions on Information Theory, vol. 45, no. 5, pp. 1456-1467, July 1999.

[8] S. Cui, A. J. Goldsmith, and A. Bahai, "Energy-constrained Modulation Optimization," IEEE Transactions on Wireless Communications, vol. 4, no. 5, pp. 2349-2360, Sept. 2005.

[9] J. G. Proakis, Digital Communications, McGraw Hill, New York, 2000.

[10] L. S. Pillutla and V. Krishnamurthy, "Energy Efficiency of Cluster based Cooperative MIMO Techniques in Sensor Networks," IEEE Transactions on Signal Processing (submitted). Available at http://www.ece.ubc.ca/ vikramk. 